\title{
Operando Magnetic Resonance Studies of Phase Behaviour and Oligomer Accumulation Within Catalyst Pores During Heterogeneous Catalytic Ethene Oligomerization
}

L. Baker ${ }^{1}$, M.P. Renshaw ${ }^{1}$, M.D. Mantle ${ }^{1}$, A.J. Sederman ${ }^{1}$, A.J. Wain ${ }^{2}$ and L.F. Gladden ${ }^{1 *}$

1. Department of Chemical Engineering and Biotechnology

University of Cambridge

Cambridge University West Site

Philippa Fawcett Drive

Cambridge CB3 0AS

U.K.

2. National Physical Laboratory

Hampton Road

Teddington TW11 0LW

U.K.

*Corresponding Author:

Lynn F. Gladden

Email: lfg1@cam.ac.uk

Submitted: 21 December 2017

Revision: 26 February 2018 


\begin{abstract}
Two-dimensional ${ }^{1} \mathrm{H}$ magnetic resonance imaging and spatially-resolved ${ }^{1} \mathrm{H}$ magnetic resonance spectroscopy and diffusion measurements were recorded as a function of time-on-stream within a fixed-bed reactor to provide direct measurements of the progress of the heterogeneous catalytic oligomerization of ethene occurring over a $1 \mathrm{wt} \% \mathrm{Ni}-\mathrm{Al}_{2} \mathrm{O}_{3}-\mathrm{SiO}_{2}$ catalyst. The catalyst bed was of internal diameter $2 \mathrm{~cm}$; magnetic resonance measurements were recorded over a bed length of 5.5 $\mathrm{cm}$. Experiments were conducted at a temperature and pressure of $110^{\circ} \mathrm{C}$ and 29 bara, respectively, with continuous downflow of ethene at a flowrate of $0.78 \mathrm{~L} \mathrm{~h}^{-1}$. During conversion the accumulation of ${ }^{1} \mathrm{H}$-containing species within the catalyst pellets was imaged, and spatially-resolved ${ }^{1} \mathrm{H}$ NMR spectra were recorded at $1 \mathrm{~mm}$ intervals along the length of the reactor. Diffusion-filtered 1D chemical shift imaging was used to discriminate between gas- and liquid-phase species along the length of the reactor at $1 \mathrm{~mm}$ intervals. Finally, spectrally-encoded pulsed field gradient measurements of molecular diffusion were employed to infer the molecular composition of the gas and liquid phases and to identify populations of these phases inside and external to the pore space of the catalyst pellets; these measurements were spatially-resolved along the length of the reactor, with data being averaged over sections of height $4 \mathrm{~mm}$. The results are consistent with oligomers of carbon number $\mathrm{C} 20$ and greater existing within the pores of the catalyst pellets which act to block the pore space, thereby deactivating the catalyst.
\end{abstract}

Keywords: operando catalysis; magnetic resonance; MRI; ethene oligomerization; heterogeneous catalysis; fixed-bed reactor 


\section{Introduction}

The application of magnetic resonance techniques to research in heterogeneous catalysis is well established. Much of this previous work has employed solid state nuclear magnetic resonance (NMR) techniques to study the chemistry and structure of heterogeneous catalysts as well as the adsorption and transport processes occurring within them [1-10]. There have been a number of approaches to studying heterogeneous catalytic processes in situ including studies under both batch and flow conditions [4], and there is increasing interest in studying heterogeneous catalytic reactions within reactor environments using magnetic resonance imaging (MRI) and NMR spectroscopy [11-19]. The motivation to implement these methods is to be able to probe both chemical composition and molecular transport in optically-opaque reactors non-invasively and without need for chemical tracers. However, the general application of magnetic resonance to studying heterogeneous catalytic conversions under operando conditions, that is, at realistic operating conditions of temperature, pressure and reactor environment, has been limited. This is due to challenges in both the construction of reactor environments built of materials compatible with operation inside a superconducting magnet, as well as obtaining spectroscopic information when spectral line broadening effects make the application of NMR spectroscopy to real catalytic conversions, in which multiple species exist within porous catalyst pellets, particularly difficult. The motivation for the present work is to explore the extent to which magnetic resonance can characterise what is happening inside the pore space of an heterogeneous catalyst in terms of the chemical composition, phase behaviour and mobility of these species. The heterogeneous catalytic ethene oligomerization reaction occurring in a fixed bed and operating at industrially-relevant conditions represents a challenging system for study but which, as demonstrated in this work, can be followed by using multiple magnetic resonance techniques in combination; namely, ${ }^{1} \mathrm{H}$ MRI, and spatially-resolved ${ }^{1} \mathrm{H}$ NMR spectroscopy, diffusion-filtered spectroscopy and spectrally-encoded measurements of molecular diffusion coefficient. Of particular interest in this study is that the gas phase feed converts through to oligomers of varying lengths which can exist in either the gas or liquid phase within the pore space of the catalyst. It is demonstrated that magnetic resonance can track reaction along the reactor and also map the amount of species in both the gas and liquid phase as well as indicate oligomer chain lengths and their evolution. The method is also able to discriminate between gas and liquid phase species confined within the pore space of the catalyst and those that are free to move within the inter-pellet space of the bed.

The catalytic oligomerization of ethene has been the subject of much academic and industrial research over the last five decades. Whilst ethene has traditionally been produced in the petrochemical industry by steam thermal cracking of hydrocarbons, alternative sources such as natural gas, coal and biomass are emerging [20]. An overview of the applications of light olefin oligomerization for the production 
of fuels and chemicals has been given by Nicholas [21] addressing both homogeneous and heterogeneous catalysts and processes. As noted by Toch and co-workers [22], whilst the homogeneous catalytic oligimerization of ethene is a well-established reaction and has been implemented at the industrial scale [23], the use of heterogeneous catalysts may help to overcome the disadvantages of using environmentally unfriendly solvents and high energy consumption, as well the relative inflexibility in tailoring product distribution characteristic of homogeneous catalytic conversions. The first report of heterogeneously catalysed dimerization of ethene was by Kimura and co-workers [24] and since then a substantial body of work has been reported. Toch et al. [22] refer to much of this work and extend it in their experimental investigation and single-event microkinetic modelling of ethene oligomerization on $\mathrm{Ni}-\mathrm{SiO}_{2}-\mathrm{Al}_{2} \mathrm{O}_{3}$. Of particular relevance to the present study is the review by Finiels et al. [20] who review the effects of pore size of the catalyst, Ni concentration, role of acid sites, and temperature and pressure of process operation on product distribution which may lie in the range of $\mathrm{C} 4-\mathrm{C} 18$ species. As noted by Finiels et al., catalyst deactivation during this conversion is a major consideration in the development of heterogeneous catalysts and catalytic processes. Deactivation is proposed to result from the strong adsorption of products on active sites and pore blocking with heavy molecules which grow from the acid-catalysed reaction of the C4-C6 product olefins which are initially formed within the catalyst pores [25-27].

The ethene oligomerization reaction therefore presents an interesting system for study and one which presents a number of measurement challenges; the products are oligomers of varying carbon number which comprise only olefinic and alkyl ${ }^{1} \mathrm{H}$ species making direct assignment of the product distribution form the acquired spectrum impossible. Moreover, the species present can exist in the gas or liquid phase. The aim of this work was to implement and demonstrate ${ }^{1} \mathrm{H}$ MRI along with spatiallyresolved spectroscopy and diffusion measurements, and the combination of these techniques, to explore the extent to which the chemical composition can be characterised and the gas- and liquidphases within the pores identified. The data show that the access to the interior of the pore space of the catalyst is lost as long chain oligomers are formed and are trapped within the pore space of the catalyst in the lower region of the bed.

\section{Experimental}

\subsection{Reactor}

All NMR experiments were performed on a super-wide bore Bruker AV-III spectrometer equipped with a $66 \mathrm{~mm}$ diameter radio-frequency (r.f.) coil operating at a ${ }^{1} \mathrm{H}$ frequency of $300.87 \mathrm{MHz}$. Figure 1 shows a schematic of the experimental continuous flow fixed-bed reactor set-up. A silicon nitride 
reactor with an internal diameter of $20 \mathrm{~mm}$ was placed within the bore of the $7.1 \mathrm{~T}$ vertical superconducting magnet. The magnetic resonance (MR) imaging region was $7 \mathrm{~cm}$ long. Thermal mass flow controllers (Bronkhorst F-201CV) were used to control the flow rate of gases into the reactor to an accuracy of $\pm 0.5 \%$. The feed lines and surface of the reactor above and below the imaging fieldof-view were covered with trace heating tape and fitted with thermocouples to control the reactor temperature. Thermocouples were also present inside the reactor, one directly above and one directly below the field-of-view, to measure the internal reactor temperature. The temperature difference across the two internal thermocouples did not exceed $2{ }^{\circ} \mathrm{C}$. Pressure control, to an accuracy of \pm 0.15 bar, was achieved through a pressure sensor at the top of the reactor and two parallel pressure control valves in the exit stream. The operation of the reactor was controlled using a LabView interface.

\subsection{Overview of Experiments}

The catalyst used was a $1 \mathrm{wt} \% \mathrm{Ni}-\mathrm{Al}_{2} \mathrm{O}_{3}-\mathrm{SiO}_{2}$ catalyst, provided by Johnson Matthey plc, in the form of cylindrical pellets of length and diameter $\sim 3 \mathrm{~mm}$; the support composition was $30 \% \mathrm{SiO}_{2}: 70 \% \mathrm{Al}_{2} \mathrm{O}_{3}$. The catalyst preparation procedure was based on that of Heveling et al. (type NiSA-1A) [28]. The surface area, pore volume and mean pore diameter of the catalyst pellets are $210 \mathrm{~m}^{2} \mathrm{~g}^{-1}, 0.53 \mathrm{~cm}^{3} \mathrm{~g}^{-1}$ and $10 \mathrm{~nm}$, respectively, as determined using nitrogen adsorption analysis. The total acid site concentration was $0.45 \mathrm{mmol} \mathrm{g}_{\text {cat }}^{-1}$, as determined by ammonia temperature programmed desorption. The mean $\mathrm{Ni}$ particle size was $5 \mathrm{~nm}$, with larger structures containing $\mathrm{Ni}, \mathrm{Si}$ and $\mathrm{O}$ (as determined from energy-dispersive X-ray spectroscopy) of diameter as large as $\sim 100 \mathrm{~nm}$ also present. $10 \mathrm{~g}$ of catalyst pellets were loaded into the cylindrical reactor of internal diameter 20 $\mathrm{mm}$, giving a bed height of $55 \mathrm{~mm}$, with $\mathrm{z}=0$ defined as the top of the catalyst bed. Spherical glass spheres of $3 \mathrm{~mm}$ diameter were packed above and below the catalyst packing to heights of $57 \mathrm{~mm}$ to aid even distribution of the feed gas and support the catalyst bed, respectively. The section of the reactor containing the pellets was positioned in the centre of the r.f. coil. The exit gas composition was measured using gas chromatography (GC) with FID detection (Agilent Technologies GC$6890 \mathrm{~N})$.

The catalyst was first dried under a flow of nitrogen gas (BOC, $99.998 \%$ purity) at $220{ }^{\circ} \mathrm{C}$ and atmospheric pressure for $12 \mathrm{~h}$. The reactor was then cooled and pressurised to the desired operating conditions for the reaction: $110{ }^{\circ} \mathrm{C}$ and 29 bar absolute. The feed gas was switched from nitrogen to ethene (BOC, $99.9 \%$ purity) flowing at $0.78 \mathrm{~L} \mathrm{~h}^{-1}$ at $110{ }^{\circ} \mathrm{C}$ and 29 bara downwards into the reactor. This corresponds to a weight-hourly-space-velocity (WHSV) of 2.15. The point at which ethene reached the catalyst bed was defined as time-on-stream $(T O S)=0 \mathrm{~h}$ and was identified by acquiring 
MR images at frequent intervals after the start of ethene flow. Reaction was monitored for $8 \mathrm{~h}$. Under these operating conditions the conversion, as determined by GC analysis of the exit gases, drops from an initial $10 \%$ to $\sim 4 \%$; the composition of the product gases remaining approximately constant over this period with mole fractions of product gases being $0.84,0.12,0.03$, and $<0.01$ for butene, hexene, octene and decene respectively.

Two further experiments were performed. First, a control experiment was performed to test for any nuclear spin relaxation time contrast effects and associated signal changes that may result from the presence of the $\mathrm{Ni}$ in the catalyst. The reactor was filled with pure support material and ethene at $110{ }^{\circ} \mathrm{C}$ and 29 bara, and the same experimental protocol was followed as for the reaction study. It was confirmed that the signal intensity of ethene at $110^{\circ} \mathrm{C}$ and 29 bara was the same in the bed packed with support only as in the bed packed with catalyst pellets, and therefore acquired signal intensities are not significantly influenced by the presence of Ni species. Second, to determine the density of ethene in the bed, the ethene signal from a deactivated bed (thereby ensuring no catalytic conversion of ethene) was compared to the signal from the same bed filled with liquid hexane; both experiments were performed at $110{ }^{\circ} \mathrm{C}$ at 29 bara. Since the density of the liquid hexane is known it is straightforward to calculate the density of ethene from the ratio of acquired signal intensities, allowing for the $\mathrm{H}: \mathrm{C}$ ratio characterising the two molecules. The deactivated bed was prepared by operating the ethene oligomerization reaction until no conversion was observed; this occurred at TOS $\sim 50 \mathrm{~h}$.

\subsection{NMR}

\subsubsection{Overview of NMR Experiments}

For a general introduction to the various NMR techniques used the reader is referred to Callaghan [29]. Implementations of these techniques as used in the study of catalysts and catalytic reactors are given elsewhere [30]. The magnetic resonance measurements employed in this work and how they are used are as follows:

Two-dimensional MRI (2D MRI) is used to map ${ }^{1} \mathrm{H}$ spin density at different spatial positions inside the reactor. As long as the ${ }^{1} \mathrm{H}$ NMR signal is free of relaxation time contrast effects, the signal intensity in the images is proportional to the number of ${ }^{1} \mathrm{H}$ nuclear spins present. Therefore, the $2 \mathrm{D}$ MRI experiment provides a spatial map of the total number of ${ }^{1} \mathrm{H}$ species present at any particular spatial location within the reactor. Given that the nuclear spin density is typically three orders of magnitude higher for a species in the liquid phase relative to the gas phase, higher signal intensities are predominantly associated with the liquid-phase species within the bed. This measurement alone does not discriminate between chemical species or characterise the amount of gas and liquid present within the reactor. 
One-dimensional chemical shift imaging (1D CSI) combines MRI and conventional spectroscopy such that ${ }^{1} \mathrm{H}$ NMR spectra are acquired at $1 \mathrm{~mm}$ intervals along the length of the reactor (taken to be the $z$-direction). For each axial location, data are averaged in the associated transverse section across the reactor for that layer thickness in $z$, therefore data come from both inside and outside the catalyst pellets. The 1D CSI data are used to estimate the average chain length along the length of the bed because these data have negligible relaxation time contrast associated with the implementation of the pulse sequence itself.

The Pulsed Field Gradient (PFG) NMR method, which is used to measure molecular displacement as a function of an observation time (also referred to as a diffusion time), is used in three different implementations in this work. The PFG NMR measurement is based on that of Stejskal and Tanner [31]. The basic principle is that the system under study is exposed to two magnetic field gradient pulses separated by an observation time $\Delta$. The attenuation of NMR signal due to molecular diffusion, characterised by the molecular diffusion coefficient, $D$, is given by:

$$
\frac{S}{S_{0}}=\exp \left(-q^{2} \Delta D\right)
$$

where $q=\gamma \delta g ; \gamma$ is the nuclear gyromagnetic ratio of the observed nuclei $\left({ }^{1} \mathrm{H}\right.$ in the present case); $\delta$ and $g$ are the duration and strength of the PFG magnetic field gradient pulses, respectively. $S$ and $S_{0}$ are the signal intensities acquired with and without implementation of the magnetic field gradients, respectively. The parameter $\Delta$ is the diffusion time between the two sets of gradient pulses. It follows that for a single-component fluid undergoing unhindered diffusion a plot of $\log \left(S / S_{0}\right)$ versus $\left(q^{2} \Delta\right)$ yields a straight line of gradient $-D$. The typical error in a PFG NMR measurement of molecular diffusion in porous materials is $3-5 \%$. If there are contributions to the total signal from several different species with different diffusivities then the signal attenuation is described by the multiexponential decay expression shown in Eq. (2):

$$
\frac{S}{S_{0}}=\sum_{i}^{N} P_{i} \exp \left(-q^{2} \Delta D_{i}\right)
$$

where $P_{i}$ is the fractional population associated with each of the diffusion coefficients $D_{i}$ and $N$ is the total number of different components, $i$.

The three different implementations are as follows. First, the PFG NMR pulse sequence was combined with the 1D CSI experiment to map molecular diffusion coefficients along the length of the reactor. This measurement provides a direct determination of the molecular diffusion coefficients along with spectral information at each spatial location. The values of diffusion coefficient are, of course, of interest in their own right and can be used in modelling heterogeneous catalytic processes. These measurements also provide insight into the molecular species (i.e., oligomer chain lengths) present in the reactor and whether a given species is in the gas or liquid phase. Further, these data are 
used to discriminate between species freely diffusing with the bed or confined within catalyst pores. Second, the spatially-resolved PFG NMR experiment was combined with the 1D CSI but using just one value of $q^{2} \Delta$, that value being chosen such that it discriminated between gas and liquid phase species in the reactor on the basis of their diffusion coefficient. This measurement will be referred to as a diffusion-filtered 1D CSI experiment; it has the advantage that it acquires data at a faster acquisition rate and with higher signal-to-noise than the full implementation of the spatially-resolved PFG experiment and can therefore track the reaction at higher temporal and spatial resolution. Third, spatially-unresolved PFG NMR measurements were acquired to measure the slowest molecular diffusion coefficients present in the reactor. This experiment was designed to give the best signal-tonoise possible to study the species moving most slowly within the reactor.

The details regarding the experimental implementation of these magnetic resonance measurements are now given.

\subsubsection{Implementation of Magnetic Resonance Experiments}

2D MRI images of the ${ }^{1} \mathrm{H}$ spin density inside the catalyst bed were acquired using a spin-echo pulse sequence [29,32], with $512 \mu$ s Gaussian-shaped slice-selective excitation and refocussing r.f. pulses. The lengths of the dephasing read and phase gradients were 0.4 and $0.3 \mathrm{~ms}$ respectively and the echo time was $2.7 \mathrm{~ms}$. For longitudinal $(z-x)$ images the data matrix consisted of $128 \times 32$ spatial points which, given a field-of-view of $64 \times 22 \mathrm{~mm}^{2}$, yields pixels with a resolution of $500 \times 690 \mu \mathrm{m}^{2}$. The slice thickness was $2 \mathrm{~mm}$. Two repetitions were acquired and the recycle delay was $5 \mathrm{~s}$ giving a total acquisition time of $5 \mathrm{~min}$. Transverse (x-y) images were acquired with a field-of-view of $22 \times 22 \mathrm{~mm}^{2}$ and a slice thickness of $2 \mathrm{~mm}$. A multi-slice imaging protocol was used to acquire axial slices separated by $6.5 \mathrm{~mm}$. The data matrix consisted of $32 \times 32$ spatial points, giving a pixel resolution of $690 \times 690 \mu^{2}$. Four averages were taken and a recycle delay of $5 \mathrm{~s}$ was used to minimise spin-lattice relaxation contrast, giving a total imaging time of $10 \mathrm{~min}$.

Implementation of 1D CSI [33] followed that of Sederman et al. [17]. The durations of the hard $90^{\circ}$ r.f. excitation pulse and the $180^{\circ}$ refocussing pulse were $60 \mu$ s and $120 \mu$ s respectively. The duration of the phase-encoding $z$-gradient was $0.3 \mathrm{~ms}$ and it was ramped from 0 to $6.9 \mathrm{G} \mathrm{cm}^{-1}$ in 64 increments giving a field-of-view in the axial $(z)$ direction of $64 \mathrm{~mm}$ and a spatial resolution of $1 \mathrm{~mm}$; the ${ }^{1} \mathrm{H}$ signal is averaged in the $x-y$ plane. The spectral width was $20000 \mathrm{~Hz}$ and the echo time was $1.4 \mathrm{~ms}$. Two repetitions were acquired and the recycle delay was $5 \mathrm{~s}$ yielding a total acquisition time of $11 \mathrm{~min}$. A recycle delay of $5 \mathrm{~s}$ was used to minimise spin-lattice relaxation contrast.

Spatially-resolved PFG NMR diffusion measurements were implemented using the 13-interval PFG stimulated echo pulse sequence [34]. The gradient pulse length, $\delta$, and the diffusion time, $\Delta$, were $2 \mathrm{~ms}$ and $300 \mathrm{~ms}$, respectively. The strength of the gradient pulse, $g$, was incremented from 0 to $77 \mathrm{G}$ 
$\mathrm{cm}^{-1}$ in 32 steps using non-linear sampling. The echo time was $4.5 \mathrm{~ms}$. To obtain spatial resolution, a phase gradient of duration $1 \mathrm{~ms}$ and incremented in 16 steps to a maximum of $0.3 \mathrm{G} \mathrm{cm}^{-1}$ was applied along the z-direction. Given the field-of-view of $64 \mathrm{~mm}$ this gave a spatial resolution of $4 \mathrm{~mm}$. For each acquisition a full free induction decay was acquired such that spectral information was extracted at each value of $q^{2} \Delta$ and for each phase gradient increment. The recycle delay was $1.3 \mathrm{~s}$ and 4 repetitions were used giving a total acquisition time of $44 \mathrm{~min}$. Preliminary study of the system showed that diffusivity values in the range $5 \times 10^{-7}-9 \times 10^{-12} \mathrm{~m}^{2} \mathrm{~s}^{-1}$ existed within the reactor. Therefore non-linear sampling was employed to improve the accuracy of the faster and slower components present. To measure diffusivities between $5 \times 10^{-7}$ and $1 \times 10^{-8} \mathrm{~m}^{2} \mathrm{~s}^{-1}$, characteristic of gas phase species, $q^{2} \Delta$ was incremented in 16 steps evenly spaced in the range $0-7 \times 10^{7} \mathrm{~s} \mathrm{~m}^{-2}$. Diffusivities between and $1 \times 10^{-8}$ and $9 \times 10^{-12} \mathrm{~m}^{2} \mathrm{~s}^{-1}$, characteristic of liquid phase species, were measured using 16 evenly spaced increments up to $q^{2} \Delta=5 \times 10^{10} \mathrm{~s} \mathrm{~m}^{-2}$. The PFG NMR data acquired for each of the two ${ }^{1} \mathrm{H}$ spectral resonances were analysed by Laplace inversion [35]. For each ${ }^{1} \mathrm{H}$ resonance, 4 distinct values of diffusion coefficient were observed and the percentage of the populations of these spins associated with each of these values of $D$ was obtained. One of the advantages of the Laplace inversion is that it is an objective, numerical process that fits distributions of molecular diffusion coefficients to the experimental data. For completeness, the data were also analysed using a non-linear regression to fit a four-component version of Eq. (2) to plots of $S / S_{0}$ vs $q^{2} \Delta$, yielding the unknown coefficients $P_{1}-P_{4}$ and $D_{1}-D_{4}$. The results were in agreement with the Laplace inversion data to within the accuracy of the non-linear regression. As discussed in Section 2.3.1 a diffusion-filtered1D CSI experiment was also implemented. A $q^{2} \Delta$ value of $3.5 \times 10^{7} \mathrm{~s} \mathrm{~m}^{-2}$ was used, which is shown in Section 3.1 to discriminate gas- and liquid-phase species. Data were acquired at a spatial resolution of $1 \mathrm{~mm}$, and the total acquisition time for these measurements was 11 min. Finally, the spatially-unresolved PFG NMR data were acquired to study the evolution of the species moving most slowly within the bed. A range of values of $q^{2} \Delta$ from 0 to $5 \times 10^{10} \mathrm{~s} \mathrm{~m}^{-2}$ were used, with 8 repetitions and a recycle delay of $5 \mathrm{~s}$, giving a total acquisition time of $21 \mathrm{~min}$.

\section{Results and Discussion}

\subsection{Identification of Gas and Liquid Phases and Composition}

Figure 2 shows a 2D spin-echo ${ }^{1} \mathrm{H}$ NMR image of the reacting catalyst bed acquired at $T O S=3.7 \mathrm{~h}$ (Fig. 2a), and 5 spectra acquired at different axial positions in the reactor using the 1D CSI measurement (Fig. 2b). In Fig. 2a, it is clearly seen that signal intensity increases with distance down the reactor. It is also noted that the higher signal intensity resides predominantly within the catalyst pellets as opposed to the space between them. The spectra shown in Fig. $2 b$ show the relative increase 
in intensity of the peak at $1 \mathrm{ppm}$ relative to that at $5 \mathrm{ppm}$ with distance down the reactor; these peaks are attributed to ${ }^{1} \mathrm{H}$ species associated with the alkyl carbons and olefinic carbons respectively. Thus, these data show that at the entrance to the bed, only ethene is present. Since all species contain one olefinic group, as the oligomer chain length grows so does the ratio of the intensity of the peak at 1 $\mathrm{ppm}$ relative to that at $5 \mathrm{ppm}$. Therefore, the spectra indicate that longer chain oligomers are present in significantly greater number as distance down the reactor increases. These data provide the motivation for the spectrally-encoded and spatially-resolved measurements of molecular diffusion which enable us to discriminate between the same species in the gas or liquid phase, and to suggest whether or not these species are confined within the pore space during the timescale of the measurement.

As a first step, discrimination of gas- and liquid- phase species is considered, along with an initial characterisation of the composition of these phases. The approach is illustrated in Fig. 3, which shows examples of spectra acquired using spectrally-encoded PFG NMR with increasing values of $q^{2} \Delta$. These data are from a $4 \mathrm{~mm}$ high section of the catalyst bed at $z=44 \mathrm{~mm}$ at $T O S=4 \mathrm{~h}$. In each case the experimental data acquired at the given value of $q^{2} \Delta$ are shown by the solid black line. The spectrum shown by the grey dotted line is the difference spectrum obtained by subtracting the spectrum acquired at each value of $q^{2} \Delta$ from the spectrum acquired for $q^{2} \Delta=0$, for which there is no diffusion weighting (i.e., attenuation). It is seen that the peak at $\sim 5 \mathrm{ppm}$ attenuates at relatively small values of $q^{2} \Delta$ consistent with the majority of species associated with the carbon-carbon double bond environment being associated with molecules characterised by fast diffusion coefficients; i.e., gasphase species. The peak at $\sim 1 \mathrm{ppm}$ attenuates far more slowly and is present through to the largest values of $q^{2} \Delta$ used, consistent with that resonance being associated with the ${ }^{1} \mathrm{H}$ species of alkyl chains of much more slowly diffusing oligomers formed during reaction. Considering Fig. $3 \mathrm{~b}$ in more detail, which was acquired at $q^{2} \Delta=3.5 \times 10^{7} \mathrm{~s} \mathrm{~m}^{-2}$, it is seen that signal associated with gas phase species has been almost completely removed due to diffusive attenuation, whereas signal from liquidphase molecules has not significantly attenuated. Thus, the spectrum shown by the grey and black lines represent the NMR signal from the gas- and liquid-phase molecules, respectively. This approach is validated by estimating the errors in this analysis by using Eq. (2), and the experimentallydetermined values of molecular diffusion coefficients and their populations and assignments to the gas or liquid phase, as reported in Table 1 . From these, it is calculated that at $q^{2} \Delta=3.5 \times 10^{7} \mathrm{~s} \mathrm{~m}^{-2},<2 \%$ of the gas phase but $>99 \%$ of the liquid phase species contribute to the acquired spectrum (black line).

It follows that for a given 'gas' or 'liquid' spectrum the ratio of the area of the spectral resonances at 1 and $5 \mathrm{ppm}$ can be used to estimate the composition of the system under study. Considering the gasphase signal, assuming the gas consists of ethene, butene, hexene and octene, the following equations hold: 


$$
\begin{gathered}
\frac{\text { area under peak at } 5 p p m}{\text { area under speak at } 1 \text { ppm }}=\frac{4 x_{e t h}+3 x_{\text {but }}+3 x_{\text {hex }}+3 x_{\text {oct }}}{5 x_{\text {but }}+9 x_{\text {hex }}+13 x_{o c t}} \\
x_{\text {eth }}+x_{\text {but }}+x_{\text {hex }}+x_{\text {oct }}=1
\end{gathered}
$$

where $x_{\text {eth }}, x_{\text {but }}, x_{\text {hex }}$ and $x_{\text {oct }}$ are the mole fractions of ethene, butene, hexene and octene respectively, and the integers identify the number of ${ }^{1} \mathrm{H}$ species contributing to each spectral peak for the given molecular species. Since for a $\operatorname{TOS}=4 \mathrm{~h}$, the GC analysis showed the gas-phase oligomerization product to be dominated by butene $\left(x_{\text {but }}=0.84\right)$, Eq. (3) and (4) are solved for the limiting case when $x_{\text {hex }} \rightarrow 0$ and $x_{\text {oct }} \rightarrow 0$ to give an estimate for the minimum value of $x_{\text {eth }}$ present in the exist gases. Taking the 'gas-phase' spectrum in Fig. 3b, it is seen that the ratio of peak areas is 9:1 which gives an estimated exit gas composition of $91 \%$ ethene and 9\% products; consistent with the GC analysis reported in Section 2.2. The low relative intensity of the peak at $1 \mathrm{ppm}$ is indicative of only short-chain gas-phase species being present and is therefore in agreement with the gas-phase composition being dominated by ethene and butane. Analysis of the liquid-phase spectrum (black line in Fig. 3b) provides an estimate of the average oligomer length. In this case, the ratio of the peak intensities at $\sim 5 \mathrm{ppm}$ relative to that at $\sim 1 \mathrm{ppm}$, gives a ratio of 3:48, which is most closely consistent with the ratio of peak intensities of 3:49 characteristic of 1-hexacosene, a C26 alpha olefin. From this analysis it is concluded that ethene and butane dominate the gas phase present, whilst a distribution of longer chain olefins, with chain lengths in excess of C20 dominate the liquid phase.

\subsection{Calculation of the Concentration of Gas and Liquid Phases Present}

The analysis in Section 3.1 shows that diffusion-filtered 1D CSI data enable discrimination of the amounts of gas and liquid phase species present in the reactor. To convert the NMR signal intensity through to a gas and liquid phase concentration, the density of representative gas and liquid phase species under the reactor operating conditions is required. A value of $756 \mathrm{~kg} \mathrm{~m}^{-3}$ for 1-hexacosene [36] is taken; given the relative insensitivity of density to carbon chain length this value is deemed representative of the liquid phase species.

Identification of the appropriate density for ethene is more challenging. With reference to Fig. 2a it is seen that at the top of the bed significant signal intensity exists inside the catalyst pellets. It is also noted that no signal intensity is seen between the catalyst pellets in the image consistent with the acquisition parameters and the operating conditions of temperature and pressure within the reactor, and the expected signal-to-noise ratio of ethene species at these conditions. Figure $2 b$ also confirms that only ethene is present in the reactor at this location, given there is negligible contribution to the lineshape at $1 \mathrm{ppm}$ (i.e. no alkyl ${ }^{1} \mathrm{H}$ species). These observations suggest that ethene is existing at a higher density inside the pore space of the catalyst than expected from the ideal gas calculation at 110 ${ }^{\circ} \mathrm{C}$ and 29 bara. This effect was observed in all experiments performed including ${ }^{1} \mathrm{H}$ NMR spectroscopy measurements in which any relaxation effects are negligible. Further, the control 
experiment described in Section 2.2 confirmed that the presence of $\mathrm{Ni}$ on the catalyst was not introducing a significant relaxation enhancement effect. It is therefore concluded that this enhancement in the acquired ${ }^{1} \mathrm{H}$ signal arises from an increase in ethene density inside the pores of the catalyst relative to its density in the inter-pellet space; i.e., the void space of the bed. The operando ethene density within the bed is obtained by comparing the MR signal intensity from the deactivated bed filled with ethene to that of the same bed filled with liquid hexane, as described in Section 2.2. Assuming that the r.f. coil response is the same for both hexane and ethene observation, and knowing the ${ }^{1} \mathrm{H}$ spin density for hexane at the reactor operating conditions, a value of $4.0 \times 10^{21}{ }^{1} \mathrm{H}$ nuclei per $\mathrm{cm}^{3}$ is obtained which equates to a mass density of $46 \mathrm{~kg} \mathrm{~m}^{-3}$; this compares with the expected value of free gas phase ethene of $26 \mathrm{~kg} \mathrm{~m}^{-3}$ at $110{ }^{\circ} \mathrm{C}$ and 29 bara calculated using the ideal gas law. Using this value for the ethene density and knowing the total void space in the reactor comprising bed voidage and catalyst porosity, which have values of $41 \%$ and $51 \%$, respectively, the concentration of gas and liquid phase species based on the total void space of the reactor is calculated. These data obtained for $T O S=4 \mathrm{~h}$ are shown in Fig. $4 \mathrm{a}$. The concentration of liquid phase species is seen to buildup significantly along the length of the reactor. This suggests that the strong signal intensity observed inside the catalyst pellets in the lower section of the bed in Fig. 2a, is associated with the build-up of liquid phase species inside the catalyst pellets. It is also observed that the concentration of gas phase species down the bed decreases slightly, consistent with the loss of access to the pore space of the catalyst as a result of the pores being blocked by the liquid-phase oligomers. This is in agreement with the ex situ measurements made by Nkosi et al. [37] who showed that spent, deactivated catalyst had a significantly reduced pore volume.

Figure $4 \mathrm{~b}$ shows the average carbon number of the liquid phase species along the length of the bed at $T O S=4 \mathrm{~h}$; these data being obtained from the diffusion-filtered 1D CSI data. The average carbon numbers should not be interpreted as suggesting that a specific oligomer chain length exists at these locations. Indeed, following the analysis of Roberts et al. [18], a Schulz-Flory product distribution [38] could be assumed given the expected reaction mechanism, where the product distribution in terms of oligomer chain length is defined as:

$W_{\mathrm{n}}=\mathrm{n}(1-\alpha)^{2} \alpha^{\mathrm{n}-1}$

where $W_{\mathrm{n}}$ is the weight fraction of products containing $\mathrm{n}$ monomer units (i.e. C2 species) and $\alpha$ is the distribution parameter, where $0 \leq \alpha \leq 1$ and can be calculated directly from an average carbon number. For this dataset at $\mathrm{z}=44 \mathrm{~mm}$ and $T O S=4 \mathrm{~h}$, a value of $\alpha=0.72$ is obtained which corresponds to a standard deviation in carbon number of $\sim 40$. This analysis suggest that the acquired data are consistent with a product distribution, whilst weighted to oligomeric products of chain length less than C20, extends to considerably longer chain lengths. However, a detailed Schulz-Flory analysis is not pursued here because such an analysis is not readily compared with the same analysis applied to the 
product stream from the bed; the MR data includes contributions from long chain oligomers held up within the pore space of the catalyst. Instead, the application of this Schulz-Flory analysis is used to illustrate that at this particular location in the bed such an analysis would show that a range of oligomer chain lengths exist and that therefore even when slow molecular diffusion coefficients are observed the NMR data do not suggest that small chain oligomers no longer exist. Small chain oligomers may exist in quite large number but their mobility will be significantly hindered by the presence of long chain oligomers.

\subsection{Identification of Chemical Species}

The measurements of molecular diffusion coefficient within the reactor are of interest in their own right and could be used as input parameters to simulation studies. In the present work, the measured diffusion coefficients are used to discriminate between gas and liquid phase species, and also enable tentative assignments of the observed diffusion coefficients to ranges of oligomer carbon numbers and provide evidence of populations of species being confined within the pore space of the catalyst.

Figure 5 shows the results of the Laplace inversion of the spatially-resolved PFG NMR data recorded at $\mathrm{z}=44 \mathrm{~m}$ for a $T O S=4 \mathrm{~h}$; a subset of which was shown in Fig. 3. As expected the spectral resonance at $5 \mathrm{ppm}$ (Fig. 5a) is associated predominantly with fast diffusion coefficients since it will be dominated by contributions from gaseous ethene and short-chain oligomers, whereas the resonance at $1 \mathrm{ppm}$ (Fig 5b) is dominated by the alkyl chain ${ }^{1} \mathrm{H}$ species and the measured distribution of diffusion is therefore dominated by longer chain species. Table 1 reports the diffusion coefficients associated with the maxima of the peaks shown in Fig. 5. To illustrate the quality of the spectrallyresolved PFG NMR data, Fig. 6 shows the actual attenuation data acquired at $\mathrm{z}=44 \mathrm{~mm}$ and $T O S=4 \mathrm{~h}$ along with the 4-component fits to these data. Data are shown for the spectral peak at $5 \mathrm{ppm}(\mathrm{a}, \mathrm{b})$ and $1 \mathrm{ppm}(\mathrm{c}, \mathrm{d})$. In each case the full $q$-range data $(\mathrm{a}, \mathrm{c})$ and the sub-set of short $q$-space data $(\mathrm{b}, \mathrm{d})$ are shown. The dashed line on each plot comes from taking the $P_{i}, D_{i}$ values from the NNLS fit of Eq. (2) to the acquired data.

With reference to Table 1, it is seen that the diffusivities of $5-6 \times 10^{-7}, 2-5 \times 10^{-10}$ and $7-8 \times 10^{-12} \mathrm{~m}^{2} \mathrm{~s}^{-1}$ are identified for species contributing to resonances at both 1 and $5 \mathrm{ppm}$. This is expected because for carbon numbers $\mathrm{C} 4+$, the molecular species will give spectral responses for both the olefinic and alkyl ${ }^{1} \mathrm{H}$ species of the same molecules, and hence will have the same diffusion coefficient. It is also seen that for each of the peaks at $1 \mathrm{ppm}$ and $5 \mathrm{ppm}$ there is one diffusion coefficient which is not in common between the two measurements; namely $\mathrm{D}=1 \times 10^{-8} \mathrm{~m}^{2} \mathrm{~s}^{-1}$ associated with the alkyl ${ }^{1} \mathrm{H}$, and $\mathrm{D}=8 \times 10^{-8} \mathrm{~m}^{2} \mathrm{~s}^{-1}$ associated with the ${ }^{1} \mathrm{H}$ bonded to the olefinic carbons. To aid the assignment of specific values of molecular diffusion coefficient to likely oligomer species, and whether they exist as 
gas or liquid phase species, the experimentally-determined self-diffusivity values of $\mathrm{C}_{10}$ to $\mathrm{C}_{60}$ nalkanes at $110{ }^{\circ} \mathrm{C}$ that were acquired by von Meerwall et al. [39] and the Fuller-Schettler-Giddings correlation [40] for estimating the diffusivity of gases were used. As shown in Figure 7, the work of von Meerwall et al. [39] reports that the diffusivity of liquid $n$-alkanes at $110{ }^{\circ} \mathrm{C}$ decreases rapidly with carbon number from $7.8 \times 10^{-9} \mathrm{~m}^{2} \mathrm{~s}^{-1}$ for $n$-octane $(\mathrm{C} 8)$ to $1.1 \times 10^{-10} \mathrm{~m}^{2} \mathrm{~s}^{-1}$ for $n$-hexacontane (C26). The values of gas phase diffusion coefficient predicted for a given molecular species by the Fuller-Schettler-Giddings correlation are adjusted for the tortuosity of the pellets, this value was taken to be 2.5 as determined by NMR diffusion measurements as described by Hollewand et al. [41]. Each of the values in Table 1 will now be discussed in turn, along with the proposed assignments of these diffusion coefficients to oligomer types, the phase in which they exist and whether or not they are confined within the pores of the catalyst.

$D=(5-6) \times 10^{-7} \mathrm{~m}^{2} \mathrm{~s}^{-1}$ : This diffusion coefficient is consistent with free self-diffusion of ethene and small $\mathrm{C}$ number oligomers (C4-C6) in the gas phase, and is therefore assigned to such species existing within the inter-pellet space of the catalyst bed. Given the small population of species characterised by this diffusion coefficient at $1 \mathrm{ppm}$, the data strongly suggest a range of C2-C6 species but with the dominant species being ethene. In support of this assignment, the Fuller-Schettler-Giddings correlation yields an ethene diffusion coefficient of $6.5 \times 10^{-7} \mathrm{~m}^{2} \mathrm{~s}^{-1}$, and $\mathrm{D}=4.4 \times 10^{-7}$ and $3.6 \times 10^{-7}$ $\mathrm{m}^{2} \mathrm{~s}^{-1}$ for butene and hexene diffusing in ethene, respectively.

(ii) $D=8 \times 10^{-8} \mathrm{~m}^{2} \mathrm{~s}^{-1}$ : This value is assigned to C2-C6 species that predominantly exist within the pore space of the catalyst during the timescale of the NMR measurement, and which are in fast exchange with a condensed gas phase within the pore space [42]. A diffusion coefficient of $8 \times 10^{-8}$ $\mathrm{m}^{2} \mathrm{~s}^{-1}$ is 6 times slower than that expected for freely-diffusing gas-phase C2-C6 species, but significantly faster than any diffusion coefficient expected for liquid-phase molecules at $110{ }^{\circ} \mathrm{C}$. These data are consistent with intra-pellet gas in dynamic exchange with a condensed gas phase in the pore space; most likely ethene in fast exchange with C4-C6 liquids condensed in the pore.

(iii) $D=1 \times 10^{-8} \mathrm{~m}^{2} \mathrm{~s}^{-1}$ : This diffusion coefficient is consistent with liquid-phase (i.e. capillary condensed) low carbon number species condensed in the pores of the catalyst. The diffusion coefficient is $\sim 50$ times slower than that expected for gases and significantly faster than liquid oligomers of higher carbon number. The observation that this $D$ value is only identified in the analysis of the alkyl peak, suggests that this is predominantly condensed butene and hexene present in the pores, since at these conditions the vapour pressure data would not predict ethene to condense into a liquid phase. Note that the population of these species is small, but this diffusion coefficient is consistently observed throughout the datasets acquired and is therefore reported and assigned.

(iv) $D=(2-5) \times 10^{-10} \mathrm{~m}^{2} \mathrm{~s}^{-1}$ : The slow diffusion coefficient and the width of this peak in the Laplace inversion data suggests an upper limit of diffusion coefficient of $1 \times 10^{-9} \mathrm{~m}^{2} \mathrm{~s}^{-1}$ and hence the existence 
of C20+ oligomers [39]. This diffusion coefficient value corresponds to a mean squared displacement of $\sim 1 \mathrm{~mm}$ in $1 \mathrm{~h}$, and is consistent with these oligomers being able to move within the bed and not trapped within the pore space of the catalyst. Note that this assignment does not uniquely assign this value of diffusion coefficient to the existence of a particular carbon number oligomer. Other shorter change oligomers will likely exist but their diffusion coefficient will be reduced in the presence of longer chain, more slowly diffusing oligomers.

(v) $D=(7-8) \times 10^{-12} \mathrm{~m}^{2} \mathrm{~s}^{-1}$ : The mean squared displacement of molecules diffusing at $8 \times 10^{-12} \mathrm{~m}^{2} \mathrm{~s}^{-1}$ for $1 \mathrm{~h}$ is $\sim 0.2 \mathrm{~mm}$, such a slow value of diffusion coefficient suggests that not only do long chain oligomers significantly in excess of C20 exist, in addition to smaller chain oligomers, but that the oligomer mixture is relatively immobile; i.e. trapped, within the pores of the catalyst. This population is therefore associated with oligomers that are blocking the pore space of the catalyst.

On the basis of these assignments it is possible to take the data shown in Fig. 4a and identify the amounts of freely moving and confined gas and liquid phase materials with the reactor. These data are shown in Fig. 8. Comparing Fig. 8a with Fig. 4b, it is seen that a decrease in pore space accessed by gas-phase species occurs as the average carbon number of the oligomers present within the bed increases.

\subsection{Evolution of Pore Blockage with Time-on-Stream}

Figure 9 shows 2D MRI acquisitions of the reacting catalyst bed. Figures 9a-c show longitudinal cross-sectional images acquired at $T O S=1.5 \mathrm{~h}, 3.7 \mathrm{~h}$ and $5.7 \mathrm{~h}$. Figure $9 \mathrm{~d}$ shows four axial crosssections from $z=13,26,39$ and $44 \mathrm{~mm}$ all acquired at TOS $=5.8 \mathrm{~h}$. As TOS increases a greater accumulation of ${ }^{1} \mathrm{H}$ signal intensity builds up within the bed, with signal intensity increasing towards the bottom of the bed. Again, it is clearly seen that the accumulation of ${ }^{1} \mathrm{H}$ signal intensity tends to be located within the catalyst pellets. The build-up of ${ }^{1} \mathrm{H}$ signal intensity, and therefore hydrogencontaining molecular species, is heterogeneous with some pellets associated with significantly greater signal intensity than others, even at the same axial location down the reactor.

Figure 10 shows the total concentration of liquid species along the length of the reactor for TOS $=0.7$, 4.0 and $5.5 \mathrm{~h}$; the data for the gas phase concentrations for these values of TOS are the same, to within experimental error, to that shown in Fig. 4a. In all cases, the concentration of liquid is seen to increase along the length of the reactor however there is a greater accumulation of liquid towards the bottom of the bed as TOS increases, consistent with Fig. 9. In Fig. 11, the diffusion coefficients characteristic of the slowly diffusing species only are shown for TOS of 0.9, 4.3 and $8.2 \mathrm{~h}$. At each TOS a bimodal distribution of diffusivities is observed. As TOS increases so does the relative amount of species 
associated with very slow diffusion coefficients $\left(\sim 10^{11}-10^{12} \mathrm{~m}^{2} \mathrm{~s}^{-1}\right)$. Although these data are not spatially-resolved and are acquired from the entire bed, the dominant contributions to the measurement will come from the regions associated with highest signal intensity in the image. Therefore this measurement is particularly sensitive to the oligomers existing within the pore space of the catalyst. These data show an increase in the slowly diffusing species trapped within the pore space of the catalyst and are therefore consistent with formation of species of increasing chain length and increasing entrapment of oligomers within the pore space; the former conclusion being supported by 1D CSI data. These data strongly support the proposal of Lallemand et al. [25-27] that rapid enrichment of liquid olefins occurs in the pores due to diffusion limitations.

\section{Conclusions}

This study has demonstrated the capability of a combination of ${ }^{1} \mathrm{H}$ MRI, spatially-resolved NMR spectroscopy, and spatially-resolved, spectrally-encoded molecular diffusion measurements to follow conversion and deactivation processes occurring during a heterogeneous catalytic conversion at reaction conditions. Alongside any insights into the particular conversion of interest, this combination of magnetic resonance methods can be applied to any heterogeneous catalytic processes which can be studied by NMR spectroscopy and this approach therefore represents an advance in our ability to screen catalysts at the bench scale and to provide data, such as molecular diffusion coefficients, that might be used in modelling reaction processes.

With respect to this particular study of the heterogeneous catalytic ethene oligomerization, spatiallyresolved ${ }^{1} \mathrm{H}$ NMR techniques have been used to provide measurements of chemical composition and molecular diffusion both as a function of spatial position inside the reactor and time-on-stream during reaction occurring over a $1 \mathrm{wt} \% \mathrm{Ni}-\mathrm{Al}_{2} \mathrm{O}_{3}-\mathrm{SiO}_{2}$ catalyst, within a fixed-bed reactor operating at a temperature and pressure of $110{ }^{\circ} \mathrm{C}$ and 29 bara, respectively, under conditions of continuous flow. By combining diffusion and spectroscopy measurements, not only can the characteristic value of molecular diffusion along the length of the reactor be identified but these values of diffusion along with the spectral analysis are used to suggest the most likely range of oligomer chain length present in the bed at any position along the length of the reactor as well as suggesting whether the species are present in the gas or liquid phase and whether or not they are trapped within the pore space of the catalyst pellet on the timescale of the MR measurements. The data are consistent with oligomers of carbon number $\mathrm{C} 20$ and greater forming within the pores of the catalyst pellets which act to block the pore space, thereby deactivating the catalyst.

\section{Acknowledgements}


The authors acknowledge the UK National Measurement System of the Department of Business, Energy and Industrial Strategy (LB and AJW) and Johnson Matthey plc (MR) for financial support. We thank Sonia Garcia and Angela Goode (Johnson Matthey plc) for catalyst preparation and TEM/EDX analysis. We also acknowledge funding towards the construction of the reactor from the European Commission in the $7^{\text {th }}$ Framework Program (GA No. 228953, OCMOL). 


\section{References}

[1] Haw, J. F., Top. Catal. 8 (1999) 81.

[2] van der Klink, J. J., Adv. in Catalysis 44 (2000) 1.

[3] M. Hunger, J. Weitkamp, Angew. Chem. Int. Ed. 40 (2001) 2954.

[4] L.F. Gladden, M. Lutecki, J. McGregor, (2012) Nuclear Magnetic Resonance Spectroscopy, in Characterization of Solid Materials and Heterogeneous Catalysts: From Structure to Surface Reactivity, Volume 1\&2 (eds M. Che and J. C. Védrine), Wiley-VCH Verlag GmbH \& Co. KGaA, Weinheim, Germany.

[5] O.B. Lapina, Catal. Today 285 (2017) 179-193

[6] D.B. Burueva, O.G. Salnikov, K.V. Kovtunov, A.S. Romanov, L.M. Kovtunova, A.K. Khudorozhkov, A.V. Bukhtiyarov, I.P. Prosvirin, V.I. Bukhtiyarov, I.V. Koptyug, J. Phys. Chem. C 120 (2016) 13541-13548.

[7] K.V. Kovtunov, O.G. Salnikov, W. Zhivonitko, I.V. Sovpin, V.I. Bukhtiyarov, I.V. Koptyug, Top. Catal. 59 (2016) 1686-1699.

[8] D. Weber, J. Mitchell, J. McGregor, L.F. Gladden, J. Phys. Chem C, 113 (2009) 6610-6615.

[9] D. Weber, A.J. Sederman, M.D. Mantle, J. Mitchell, L.F. Gladden, Phys. Chem. Chem. Phys. 12 (2010) 2619-2624.

[10] C. D’Agostino, G.L. Brett, P.J. Miedziak, D.W. Knight, G.J. Hutchings, L.F. Gladden, M.D. Mantle, Chem. Eur. J. 18 (2012) 14426 - 14433.

[11] E.H.L. Yuen, A.J. Sederman, L.F. Gladden, Appl. Catal. A: Gen. 232 (2002) 29-38.

[12] L.F. Gladden, P. Alexander, M.M. Britton, M.D. Mantle, A.J. Sederman, E.H.L. Yuen, Magn. Reson. Imaging 21 (2003) 213-219.

[13] B.S. Akpa, M.D. Mantle, A.J. Sederman, L.F. Gladden, L.F., Chem. Commun. (2005) 27412743.

[14] N.L. Nguyen, R. Reimert, E.H. Hardy, Chem. Eng. Technol. 29 (2006) 820-827.

[15] L.F. Gladden, F.J.R. Abegão, C.P. Dunckley, D.J. Holland, M.H. Sankey, A.J. Sederman, Catal. Today 155 (2010) 157-163.

[16] I. Koptyug, A.A. Lysova, A. Kulikov, V.A. Kirrillov, V.N. Parmon, R.Z. Sagdeev, Appl. Catal. A: Gen. 267 (2004) 143-148.

[17] A.J. Sederman, M.D. Mantle, C.P. Dunckley, Z.Y. Huang, L.F. Gladden. Catal. Lett. 103 (2005) $1-8$. 
[18] S.T Roberts, M.P. Renshaw, M. Lutecki, J. McGregor, A.J. Sederman, M.D. Mantle, L.F. Gladden, Chem. Commun. 49 (2013) 10519-10521.

[19] J. Ulpts, W. Dreher, M. Klink, J. Thöming, Appl. Catal. A: Gen. 502 (2015) 340-349.

[20] A. Finiels, F. Fajula, V. Hulea, Catal. Sci. Technol. 4 (2014) 2412-2426.

[21] C.P. Nicholas, Appl. Catal. A: Gen. 543 (2017) 82-97

[22] K. Toch, J.W. Thybaut, G.B. Marin, Appl. Catal. A: Gen. 489 (2015) 292-304.

[23] Ullmann's Encyclopedia of Industrial Chemistry (2014) Wiley-VCH.

[24] K. Kimura, H. Ai, A. Ozaki, J. Catal. 18 (1970) 271-280.

[25] M. Lallemand, A. Finiels, F. Fajula, V. Hulea, Appl. Catal. A301 (2006) 196-201.

[26] M. Lallemand, O.A. Rusu, E. Dumitriu, A. Finiels, F. Fajula, V. Hulea, Appl. Catal. A338 (2008) 37-43.

[27] M. Lallemand, A. Finiels, F. Fajula, V. Hulea, Chem. Eng. J. 172 (2011) 1078-1082.

[28] J. Heveling, C.P. Nicolaides, M.S. Scurrell, Appl. Catal. A173 (1998) 1-9.

[29] P.T. Callaghan, Principles of Nuclear Magnetic Resonance Microscopy, Oxford University Press, Oxford (1993).

[30] L.F. Gladden, M.D. Mantle, A.J. Sederman, Adv. Chem. Eng, 30 (2005) 63-135.

[31] E.O. Stejskal, J.E. Tanner, J. Chem. Phys. 42 (1965) 288-292.

[32] A.J. Sederman, L.F. Gladden, Chem. Eng. Sci., 56 (2001) 2615-2628.

[33] A.A. Maudsley, S.K. Hilal, W.H. Perman, H.E. Simon, J. Magn. Reson. 196951 (1983) 147152.

[34] R.M. Cotts, M.J.R. Hoch, T. Sun, J.T. Markert, J. Magn. Reson. 83 (1989) 252-266.

[35] K.G. Hollingsworth, M.L. Johns, J. Colloid Interface Sci. 258 (2003) 383-389.

[36] D.-Y. Peng, D.B. Robinson, Ind. Eng. Chem. Fundamen. 15 (1976) 59-64.

[37] B. Nkosi, F.T.T. Ng, G.L. Rempel, Appl. Catal. A161 (1997) 153-166.

[38] J. Skupińska, Chem. Rev. 91 (1991) 613-648.

[39] E. von Meerwall, S. Beckman, J. Jang, W.L. Mattice, J. Chem. Phys. 108 (1998) 4299-4304.

[40] E.N. Fuller, P.D. Schettler, J.C. Giddings, Ind. Eng. Chem. 58 (1966) 18-27.

[41] M.P. Hollewand, L.F. Gladden, Chem. Eng. Sci. 50 (1995) 309-326.

[42] R. Valiullin, P. Kortunov, J. Kärger, V. Timoshenko, J. Chem. Phys. 120 (2004) 11804-11814. 


\section{Tables}

Table 1

\begin{tabular}{|c|c|c|c|c|c|}
\hline \multicolumn{2}{|c|}{ peak at $1 \mathrm{ppm}$} & \multicolumn{2}{|c|}{ peak at $5 \mathrm{ppm}$} & \multirow{2}{*}{ species present } & \multirow{2}{*}{ phase } \\
\hline$D_{i}\left[\mathrm{~m}^{2} \mathrm{~s}^{-1}\right]$ & $P_{i}[-]$ & $D_{i}\left[\mathrm{~m}^{2} \mathrm{~s}^{-1}\right]$ & $P_{i}[-]$ & & \\
\hline $5 \times 10^{-7}$ & 0.06 & $6 \times 10^{-7}$ & 0.64 & $\begin{array}{l}\text { short chain alkenes } \\
\text { (C2 -C6) }\end{array}$ & $\begin{array}{l}\text { free gas in inter- } \\
\text { particle space of } \\
\text { reactor }\end{array}$ \\
\hline & & $8 \times 10^{-8}$ & 0.29 & $\mathrm{C} 2-\mathrm{C} 6$ & $\begin{array}{l}\text { intra-pellet gas in } \\
\text { dynamic } \\
\text { exchange with } \\
\text { condensed phase } \\
\text { in pore space }\end{array}$ \\
\hline $1 \times 10^{-8}$ & 0.05 & & & $\mathrm{C} 4, \mathrm{C} 6$ & $\begin{array}{c}\text { condensed in pore } \\
\text { space }\end{array}$ \\
\hline $2 \times 10^{-10}$ & 0.38 & $5 \times 10^{-10}$ & 0.03 & $\begin{array}{l}\text { range of C number } \\
\text { oligomers, including } \\
\text { presence of long chain } \\
\text { oligomers }(\mathrm{C} 20+)\end{array}$ & $\begin{array}{l}\text { liquid in inter- } \\
\text { pellet space }\end{array}$ \\
\hline $7 \times 10^{-12}$ & 0.51 & $8 \times 10^{-12}$ & 0.04 & $\begin{array}{l}\text { range of C number } \\
\text { oligomers, including } \\
\text { presence of long chain } \\
\text { oligomers }(\mathrm{C} 20+)\end{array}$ & $\begin{array}{l}\text { liquid trapped in } \\
\text { catalyst pores }\end{array}$ \\
\hline
\end{tabular}




\section{Figure Captions}

\section{Figure 1}

Schematic of the reactor set-up: mass flow controllers (1); liquid feed vessel (2); high pressure liquid chromatography (HPLC) pump (3); reactor (4); NMR magnet (5); liquid knock-out pot (6). PI identifies the pressure indicator.

\section{Figure 2}

(a) Longitudinal-plane $(z-x)$ 2D MRI image acquired during the ethene oligomerization reaction. Data are shown for $T O S=3.7 \mathrm{~h}$. The image slice thickness is $2 \mathrm{~mm}$, and the in-plane spatial resolution is $500 \mu \mathrm{m}(z) \times 690 \mu \mathrm{m}(x)$. High signal intensity (lighter shades) indicates the presence of larger amounts of ${ }^{1} \mathrm{H}$ species. (b) Five spectra taken from the ${ }^{1} \mathrm{H}$ 1D CSI dataset acquired at TOS $=3.6 \mathrm{~h}$. Each spectrum is acquired from the $1 \mathrm{~mm}$ thick transverse slice section through the bed identified by the dotted lines in (a). ${ }^{1} \mathrm{H}$ chemical shifts are referenced to the ${ }^{1} \mathrm{H}$ resonance of tetramethylsilane (TMS).

\section{Figure 3}

${ }^{1}$ H NMR spectra acquired during a spatially-resolved PFG NMR diffusion measurement. Data are shown for a slice at a position $z=44 \mathrm{~mm}$ from the entrance to the catalyst bed at $T O S=4 \mathrm{~h}$. The solid black lines show the data acquired for acquisition parameter values of $q^{2} \Delta=$ (a) 0 , (b) $3.5 \times 10^{7}$, (c) $5.1 \times 10^{10} \mathrm{~s} \mathrm{~m}^{-2}$. The dotted grey lines in each figure show the difference between the acquired data and the unattenuated spectrum at $q^{2} \Delta=0$.

\section{Figure 4}

Characterisation of phase behaviour and evolution of average oligomer chain length down the reactor at $T O S=4 \mathrm{~h}$. (a) Concentration of liquid- (solid line) and gas- (dashed line) phase species. (b) The average carbon number of the liquid-phase species.

\section{Figure 5}

Probability distribution of diffusivities obtained by Laplace inversion of the spatially-resolved PFG NMR diffusion data recorded from a slice thickness of $4 \mathrm{~mm}$ located at position $z=44 \mathrm{~mm}$ at $T O S=4 \mathrm{~h}$. Data are shown for the spectral peak at (a) $5 \mathrm{ppm}$, and (b) $1 \mathrm{ppm}$ relative to TMS. 


\section{Figure 6}

Example of spatially-resolved PFG NMR diffusion measurements. Data (0) are shown at position $z=44 \mathrm{~mm}$ at $T O S=4 \mathrm{~h}$; the signal attenuation as a function of $q^{2} \Delta$ for the spectral peaks at $5 \mathrm{ppm}$ and $1 \mathrm{ppm}$ are shown in $(\mathrm{a}, \mathrm{b})$ and $(\mathrm{c}, \mathrm{d})$, respectively. Both the full range of $q^{2} \Delta$ is shown (a,c) and a shorter range $q^{2} \Delta<1 \times 10^{8} \mathrm{~s} \mathrm{~m}^{-2}(\mathrm{~b}, \mathrm{~d})$. The dashed lines shows the fits the NNLS fit of Eq. (2) to the acquired data. As discussed in the text this analysis gave values of ${ }^{1} \mathrm{H}$ spin population and molecular diffusivities in agreement with the data obtained from the Laplace inversion (Fig. 5) reported in Table 1.

\section{Figure 7}

Experimentally-determined diffusivity values of C8-C60 n-alkanes at $110{ }^{\circ} \mathrm{C}$ [39].

\section{Figure 8}

Concentration of (a) gas phase, and (b) liquid phase species along the length of the bed at TOS $=4 \mathrm{~h}$. For each phase, the concentration of species freely moving with the bed $(\bullet)$ and that which is confined with the pore ace ( ) is identified.

\section{Figure 9}

(a)-(c) Longitudinal (z-x) 2D MRI images of the catalyst bed at TOS of (a) $1.5 \mathrm{~h}$, (b) $3.7 \mathrm{~h}$, (c) $5.7 \mathrm{~h}$. The image slice thickness is $2 \mathrm{~mm}$, and the in-plane spatial resolution is $500 \mu \mathrm{m}(z) \times$ $690 \mu \mathrm{m}(x)$. High signal intensity (lighter shades) indicates the presence of larger amounts of ${ }^{1} \mathrm{H}$ species. (d) Transverse $(x-y)$ 2D MRI images with slice thickness $=2 \mathrm{~mm}$, and in-plane spatial resolution $690 \mu \mathrm{m}(x) \times 690 \mu \mathrm{m}(z)$. Images are shown from a dataset recorded at TOS = $5.6 \mathrm{~h}$, and are taken from positions in the bed at 13,26,39 and $44 \mathrm{~mm}$ in the bed as indicated in the longitudinal image recorded at TOS $=5.7 \mathrm{~h}$. Signal intensity in all the images has been normalised relative to the intensity of the brightest pixel in (d).

\section{Figure 10}

Concentration of liquid-phase species along the length of the reactor at $T O S=0.7,4.0$ and $5.5 \mathrm{~h}$. 


\section{Figure 11}

Probability distributions of molecular diffusion coefficients obtained by Laplace inversion of spatially-unresolved PFG NMR measurements at TOS=0.9,4.3 and 8.2 h. Only slowly diffusing species are shown. Although spatially-unresolved these data will be dominated by the regions of the bed containing most ${ }^{1} \mathrm{H}$ species; such species are predominantly towards the bottom of the reactor as seen in Figures 2 and 9. 


\section{Table Captions}

\section{Table 1}

Parameters from the Laplace inversion of the spatially-resolved, spectrally-encoded PFG NMR data recorded at $z=44 \mathrm{~mm}$ and $T O S=4 \mathrm{~h}$. 


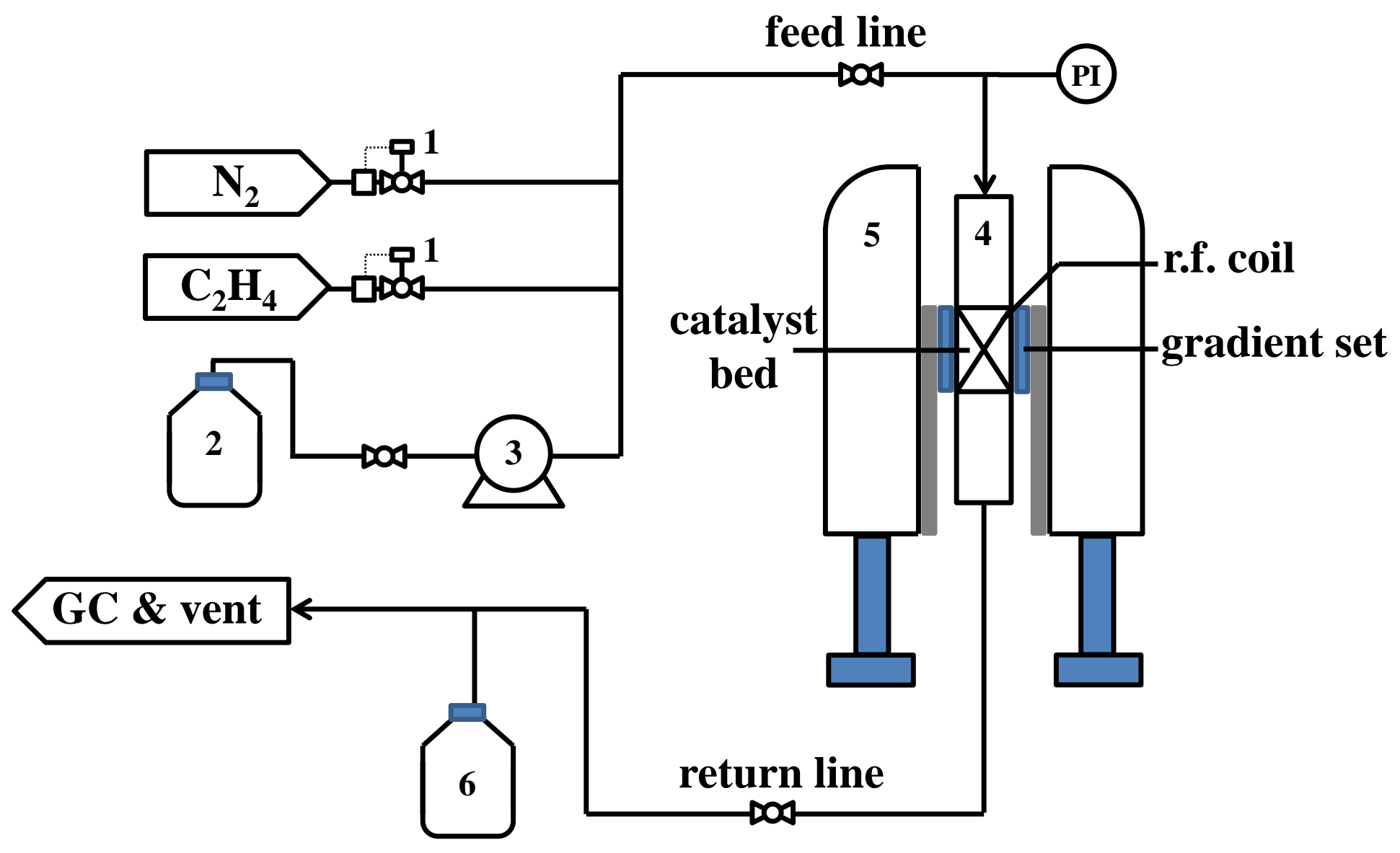

Figure 1 


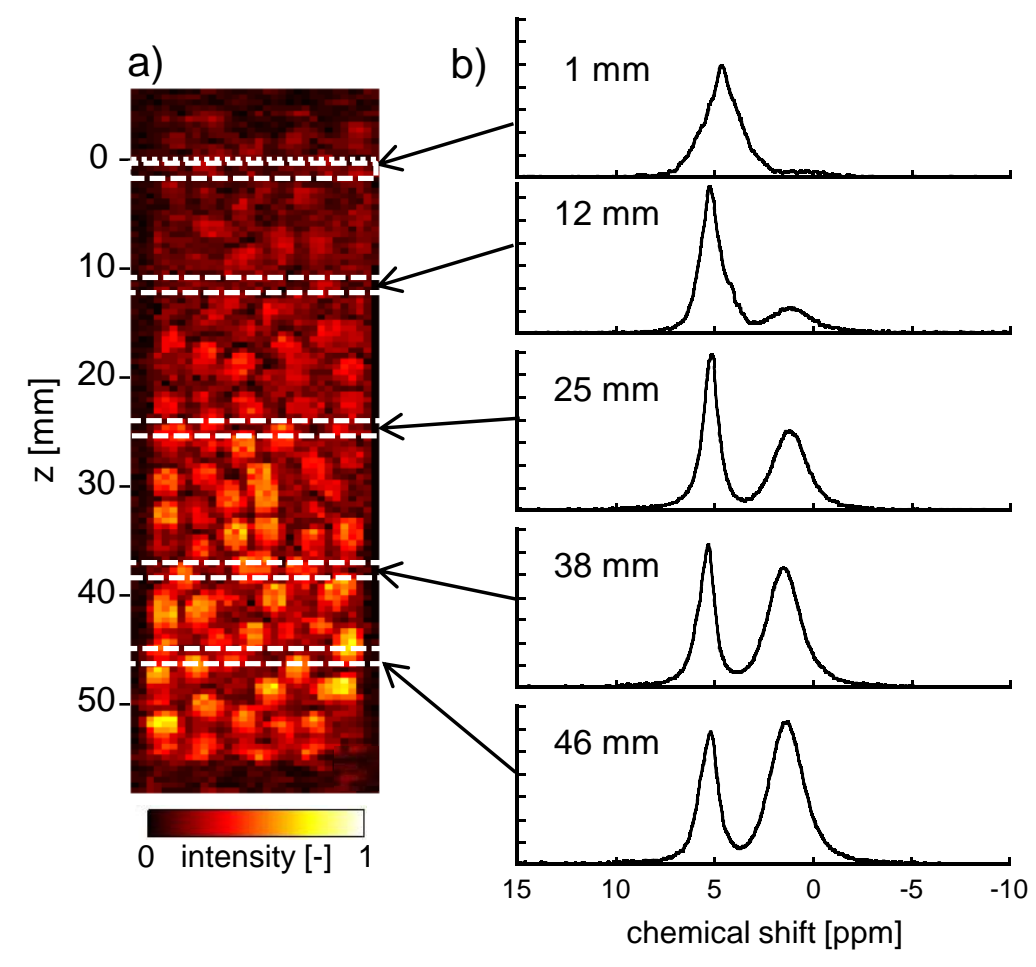

Figure 2 


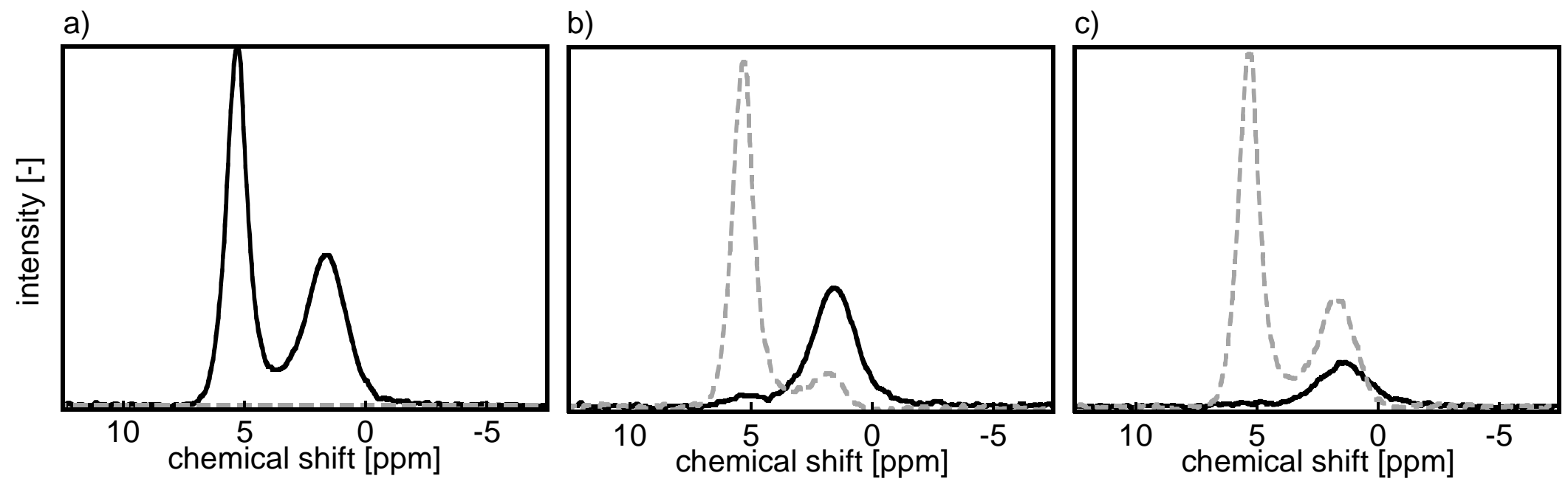

Figure 3 

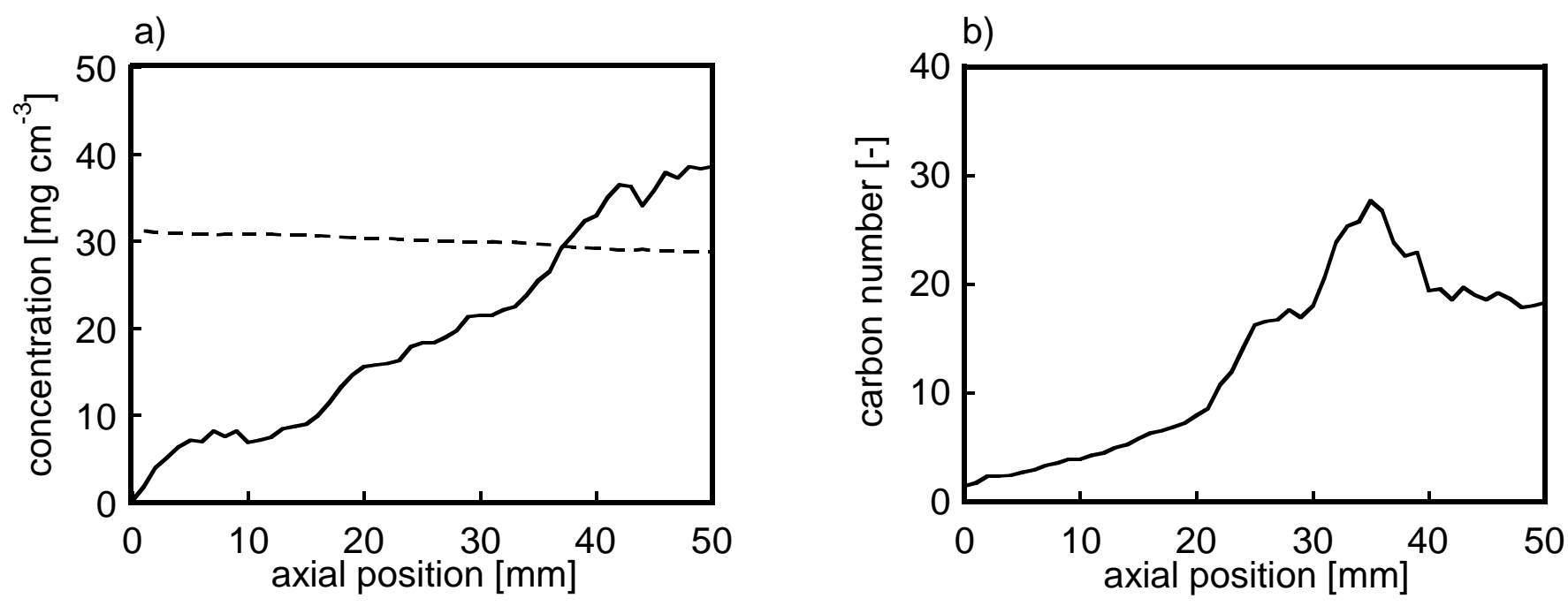

Figure 4 

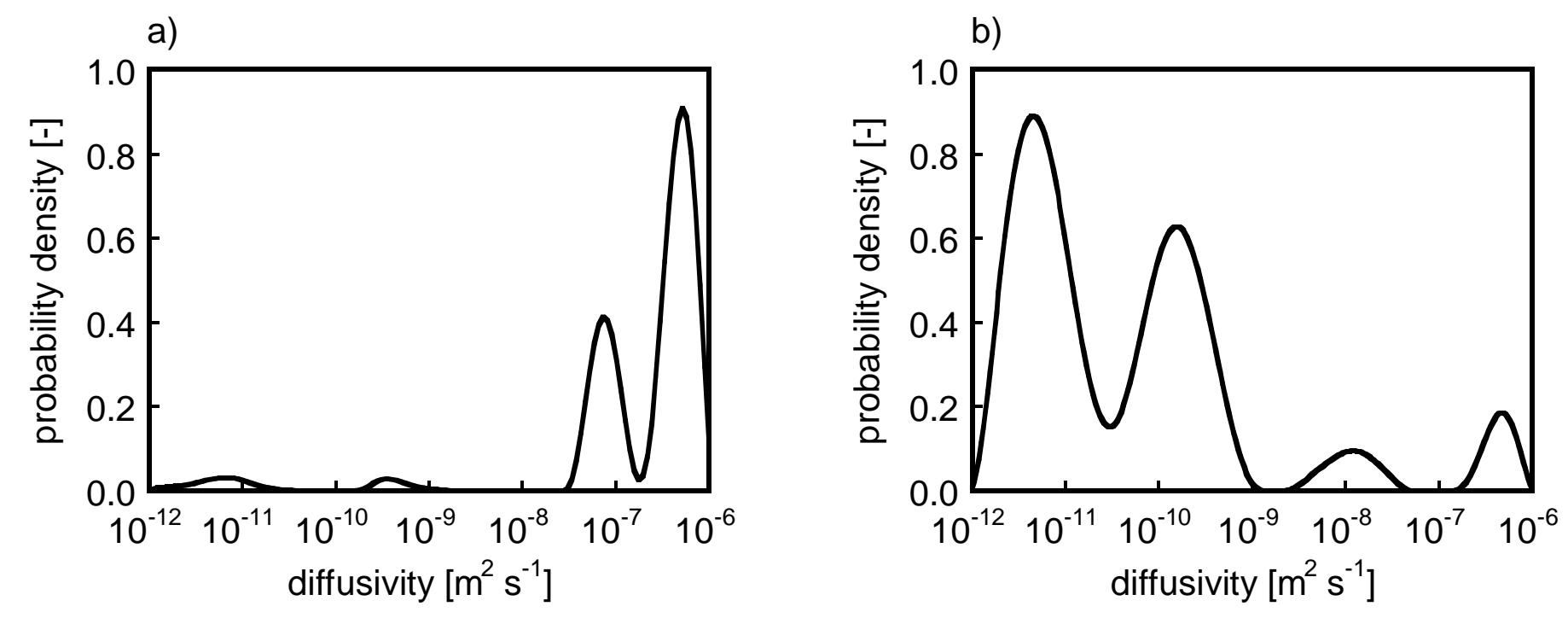

Figure 5 
a)

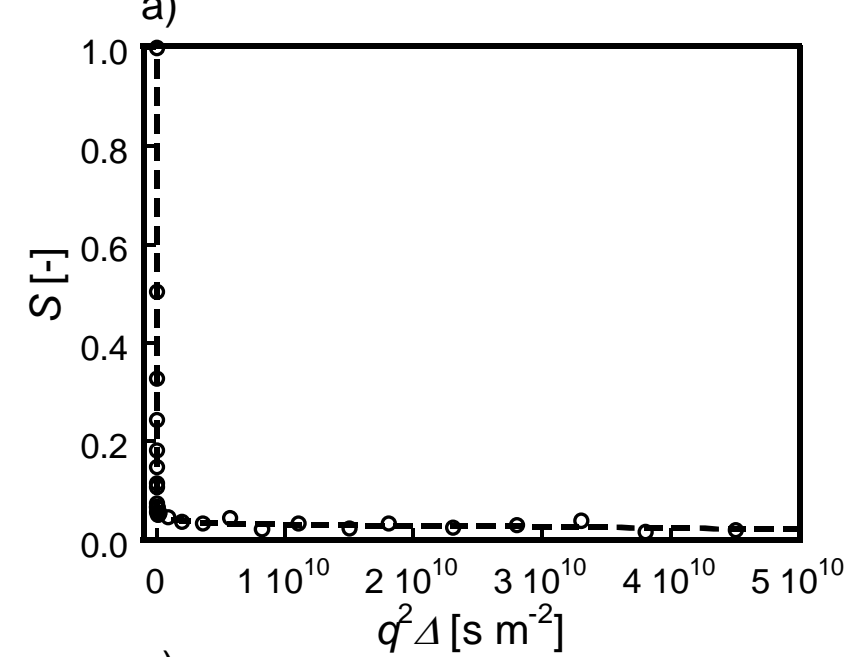

c)

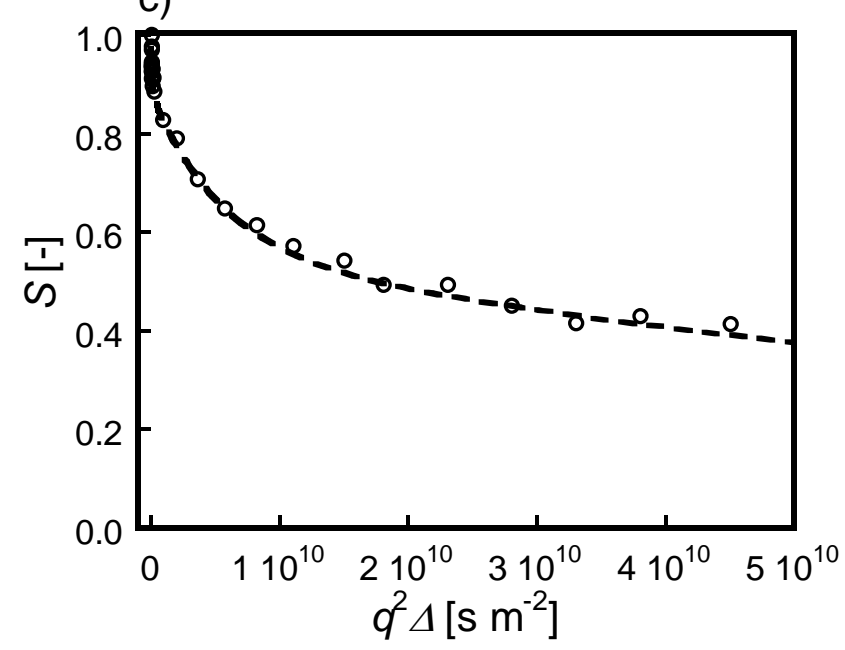

b)
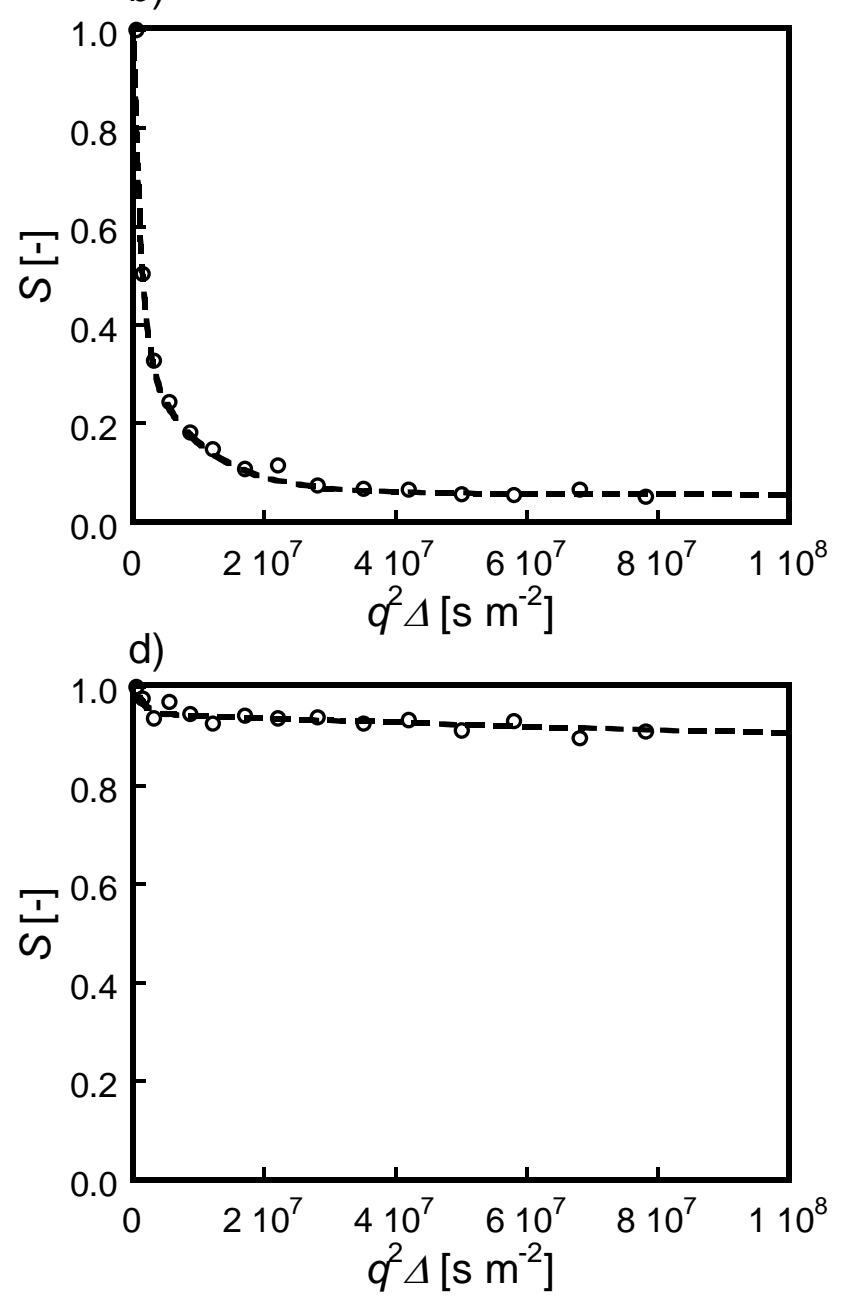

Figure 6 


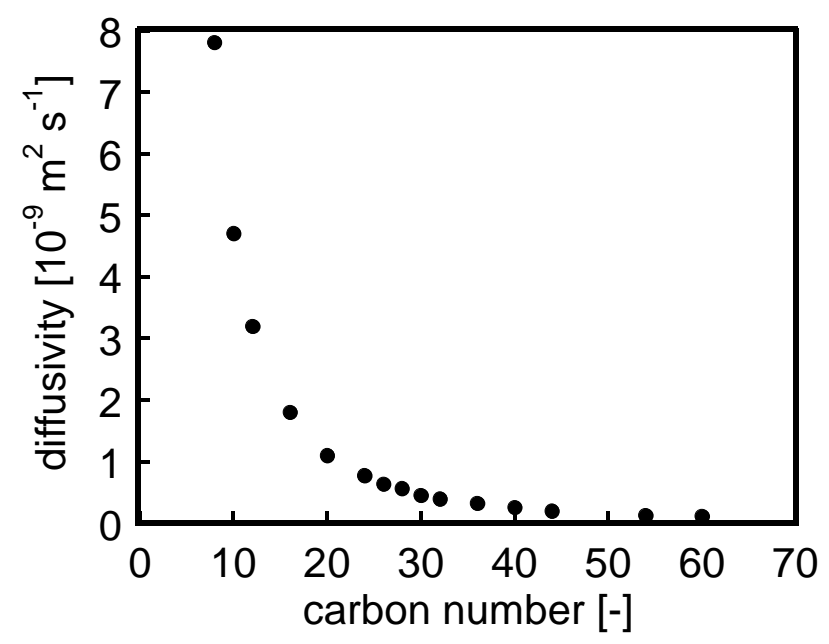

Figure 7 
a)

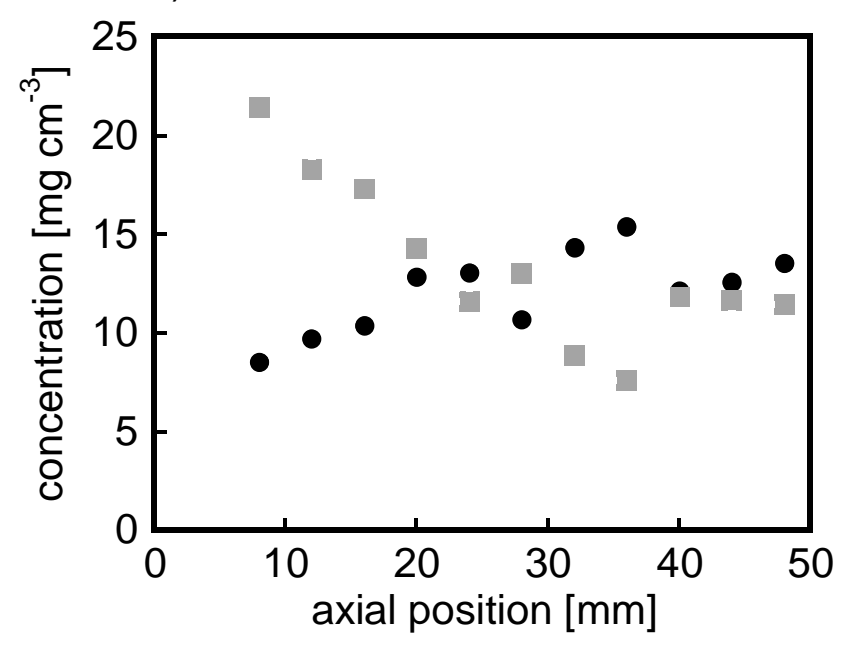

b)

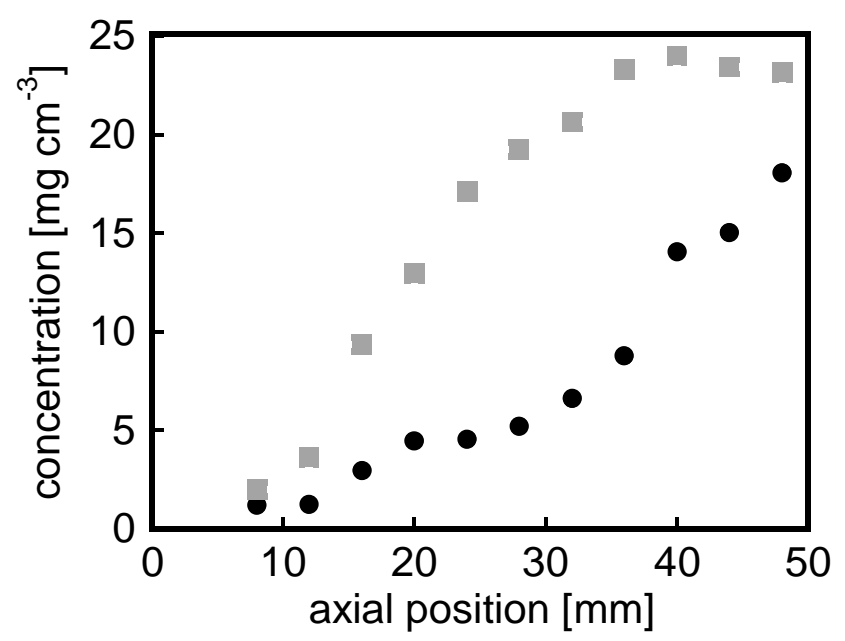

Figure 8 


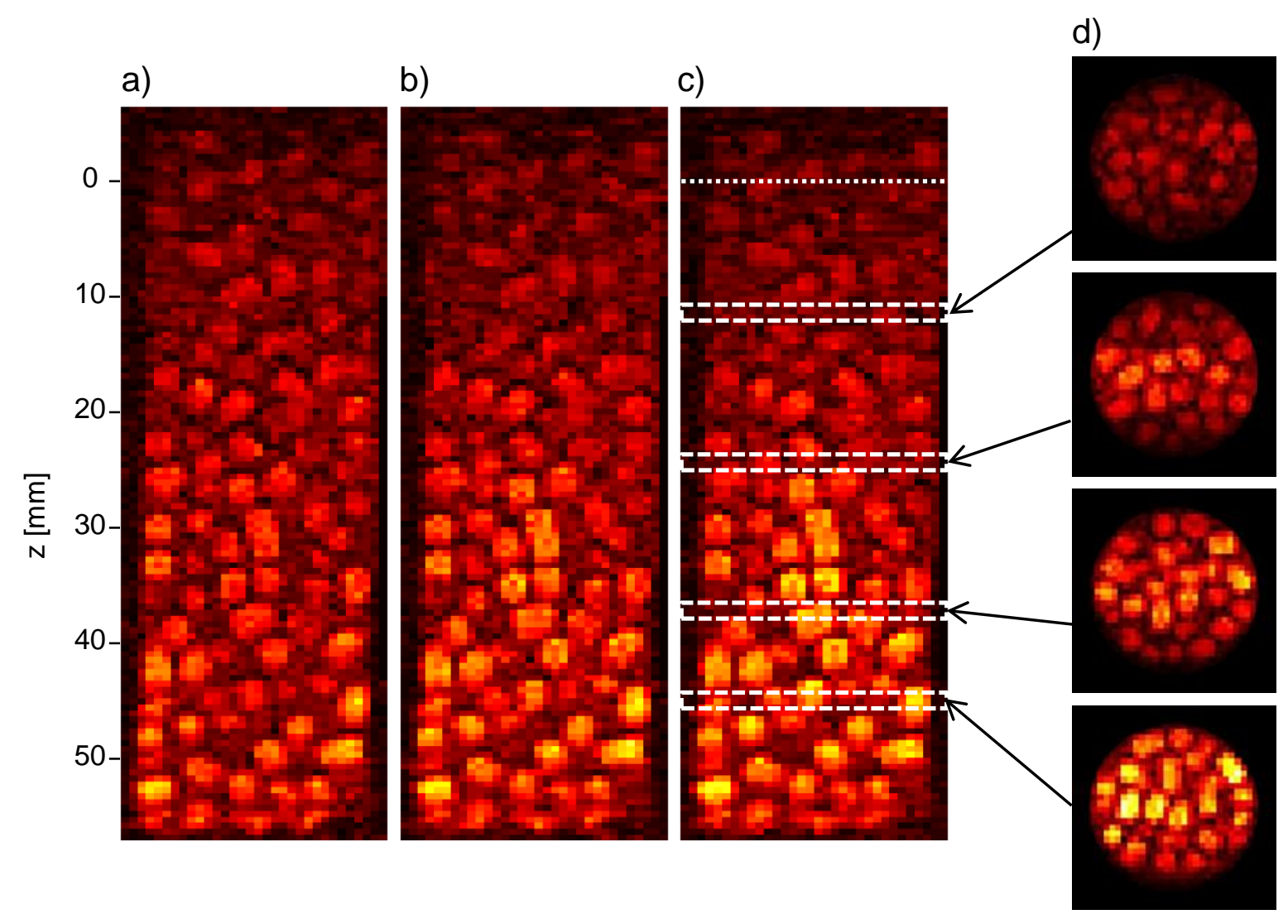

Figure 9 


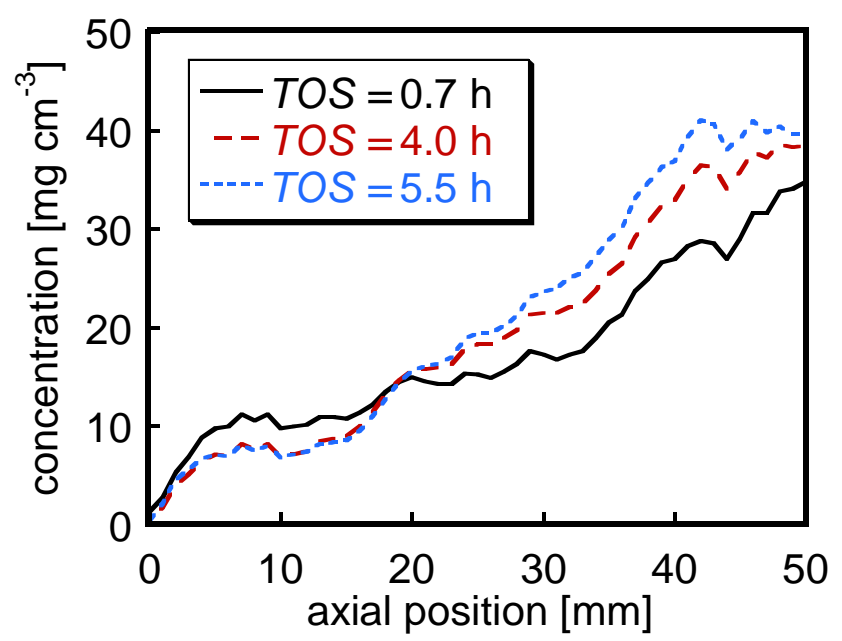

Figure 10 


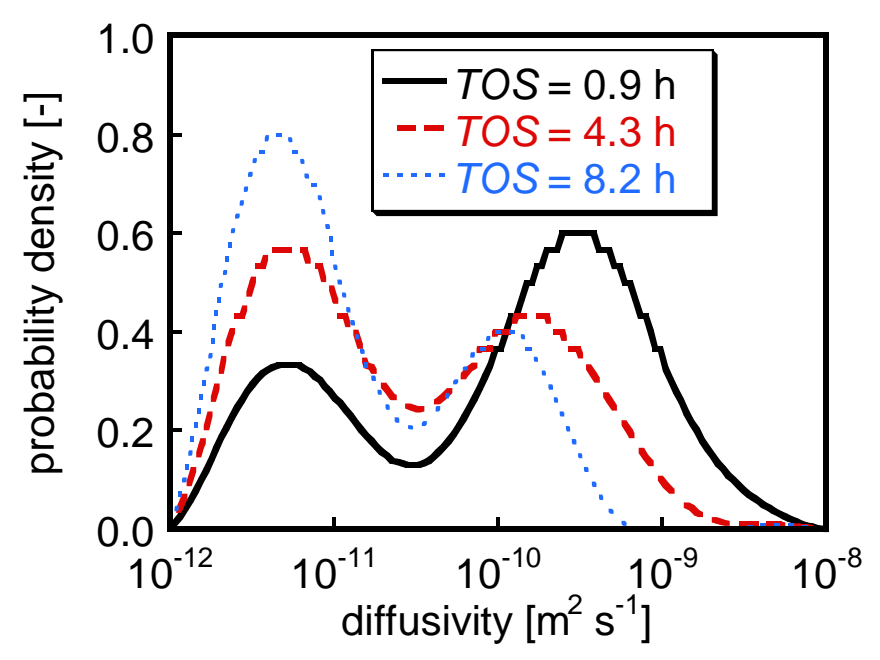

Figure 11 